\title{
A problem in determining fitness differences
}

\author{
FRANK B. LIVINGSTONE
}

Department of Anthropology, University of Michigan, Ann Arbor, MI 48109

Although differential contributions to the next generation have frequently been used to measure differences in fitness, at genetic equilibrium there will be no such differences among genotypes with balanced polymorphism. For a single locus the frequencies, after selection, of the three genotypes, $A A, A a$, aa, will be: $W_{11} p^{2} / W, 2 W_{12} p q / \bar{W}$ and $W_{22} q^{2} / \bar{W}$, where

$$
\bar{W}=W_{11} p^{2}+2 W_{12} p q+W_{22} q^{2} .
$$

To estimate the contribution of any genotype to the next generation, the average fitness of the offspring can be calculated from the frequencies of possible matings for that genotype and the average fitness of the offspring of each mating. For the $A A$ genotype the average fitness of the offspring will be

or $\quad \bar{W}^{-1}\left(W_{11} p\left[W_{11} p+W_{12} q\right]+W_{12} q\left[W_{12} p+W_{22} q\right]\right)$.

$$
\left(W_{11} p^{2} / \bar{W}\right) W_{11}+\left(2 W_{12} p q / \bar{W}\right)\left(0.5 W_{11}+0.5 W_{12}\right)+\left(W_{22} q^{2} / \bar{W}\right) W_{12}
$$

At genetic equilibrium, $W_{11} p+W_{12} q=W_{12} p+W_{22} q=\bar{W}$, so that average fitness will be simply $\bar{W}$. For the $A a$ genotype, the average fitness of the offspring will be

$$
\begin{aligned}
\left(W_{11} p^{2} / \bar{W}\right)\left(0.5 W_{11}+0.5 W_{12}\right)+\left(2 W_{12} p q / \bar{W}\right)\left(0.25 W_{11}+0.5 W_{12}+0.25 W_{22}\right) & +\left(W_{22} q^{2} / \bar{W}\right)\left(0.5 W_{12}+0.5 W_{22}\right),
\end{aligned}
$$

which also reduces to $\bar{W}$; and similarly for the $a a$ genotype. This conclusion is only valid when selection occurs as early mortality. However, the sickle cell gene appears to be close to equilibrium in many populations in Africa; and since most selection due to either sickle-cell anaemia or cerebral malaria occurs in early childhood, this may explain the inconclusive nature of the data on differences in the average number of surviving offspring for sicklers and non-sicklers. 